\title{
The tension between efficiency and effectiveness: a study of dietetic practice in primary care
}

\begin{abstract}
Background: Primary health care dietitians have a vital role to play in the prevention and management of chronic disease. Working in primary care requires efficient and effective management of practice to ensure client and practitioner needs are met. The aim of this study was to explore the way in which primary care dietitians in Australia view the constructs of efficiency and effectiveness within the context of their practice.

Methodology: This study used an exploratory qualitative design within a pragmatist framework. Individual semi-structured telephone interviews were conducted with Australian primary care dietitians. All interviews were audio-recorded, transcribed verbatim and analysed using an inductive thematic approach.
\end{abstract}

Results: Twenty dietitians (17 females) working as private practitioners in primary care from three Australian states participated in this study. Three themes emerged from the data. The first theme revealed that seeking efficiency and especially effectiveness were important to primary care dietitians and that there was a tension between the two. The second theme identified that efficiency and effectiveness are influenced by personal and structural factors. The final theme explored how dietitians are actively seeking ways to be more efficient and effective, including supportive networks, and the utilisation of technology.

Conclusion: Achieving a balance between efficiency and effectiveness in primary care dietetics is challenging to practitioners, who may require further training and support to enhance productivity, time management and resource utilisation. Structured issues exist for the workface. Further studies are required to quantify these findings and to explore whether it is possible to optimise efficiency and effectiveness and achieve sustainability of the dietetic workforce in primary care.

Key words: primary health care, private practice, dietetics, nutritionist, outcome assessment (health care), sustainability 
Dietitians are a valued component of the primary health care workforce. Dietetic input can assist patients to significantly improve chronic disease health markers beyond usual care ${ }^{(1,2)}$. A study of patients with type 2 diabetes in the USA found that for every consultation with a dietitian in primary care, there was an associated decrease of 4.7 hospital admissions per 100 person-years ${ }^{(3)}$. Dietetic input into primary care thus has clear economic benefits ${ }^{(1,3)}$. A New Zealand study estimated that every $\$ 1$ allocated to dietetic interventions had the potential to save \$5.50-\$99 in total health care costs due to improved health and productivity gains ${ }^{(1)}$. Given that one in every four adults suffer from multiple chronic diseases in developed countries such as the USA, Australia and UK ${ }^{(4,5,6)}$, the demand for dietetic consultations in primary care is increasing ${ }^{(7)}$.

Working in primary care requires efficient and effective management of practice ${ }^{(8,9)}$. While there has been a strong emphasis on evidence-based practice to promote effectiveness ${ }^{(10-15)}$, less has been written about efficiency ${ }^{(7)}$, or how these two concepts operate in the primary care setting. Effectiveness describes the degree to which something is successful in producing a desired result ${ }^{(16)}$. Efficiency refers to the degree to which a task can be achieved with minimal time, effort and resources, and is an important factor in business success ${ }^{(17)}$. Primary care dietitians in Australia comprise $29 \%$ of the dietetic workforce and are typically small business owners or contractors ${ }^{(7)}$. Despite the size of the workforce, little is known about the potential influencers of efficiency or the impact it has on maintaining effectiveness in providing nutrition care.

The few studies reporting on the dietetic primary care workforce have revealed challenges to providing best practice within the primary care setting, including being able to achieve worklife balance, receiving inadequate remuneration secondary to excessive unbillable hours required for administration tasks, and insufficient consultation allocations by funding agencies ${ }^{(18-20)}$. Previous studies have hypothesised that one way in which dietitians in this setting cope with these constraints is to provide "abbreviated care" to overcome challenges associated with lack of sufficient remuneration for time spent ${ }^{(21,22)}$. Abbreviated care has been described as a less holistic approach and more succinct method of treatment through the simplification of the intended outcome ${ }^{(21)}$, which may compromise patient care. Other methods of optimising 
Understanding how efficiency and effectiveness are perceived in primary care dietetic practice is important in identifying how this workforce can be better prepared and supported in their role. A pragmatist approach was employed, allowing the focus to be on achieving outcomes that are relevant to the stakeholders, and able to directly impact on practice ${ }^{(23)}$. The aim of this study was to explore the way in which primary care dietitians in Australia view the construct of efficiency and effectiveness in the context of their practice.

\section{Methods}

This study utilised an exploratory qualitative design. A qualitative approach is useful for investigating a topic that has been relatively unstudied ${ }^{(24)}$. Individual semi-structured telephone interviews were used to collect data on dietitians' perceptions of efficiency and effectiveness in primary care practice. Data were collected in parallel with a related study, investigating the use of technology in the same target group. The study was approved by [removed for blind peer review] (Institutional Ref No: 2017/033).

Potential participants were Accredited Practising Dietitians (APDs) in Australia, currently working in primary care (self-employed or working for others). Key demographic and workplace variables such as gender, years since graduation, experience in primary care, geographical location of employment and primary clientele (for example, sports, weight management, chronic disease) were collected to monitor diversity throughout recruitment. Recruitment was conducted in an online format, using a combination of convenience and snowball sampling to enhance the likelihood of national participation. Convenience sampling methods included ${ }^{(24)}$ circulating recruitment materials nationally through email newsletters from Dietitian Connection $₫$ (professional education and networking platform for Australian dietitians), the (removed for blinding) University Nutrition \& Dietetics Facebook page, a primary care dietetics email group, and through the researchers' networks. An information form detailing a description of the study and an advertisement poster were provided, both containing a link to an online survey to collect names and contact information of dietitians who were willing to participate. Participants were encouraged to forward recruitment materials on to other potential participants within their networks, a form of snowball sampling ${ }^{(24)}$. Due to the method of recruitment, the exact number of dietitians who received the study invitation is unknown. 
A semi-structured interview protocol was developed based on a review of the literature. The protocol commenced by exploring effectiveness and efficiency (for this study) and then technology (for the related study). The protocols for the two studies were administered in a single interview session by the same interviewer. The draft protocol was pilot-tested with a primary care dietitian and modified accordingly, with minor changes made to ensure questions were open and face validity was achieved. An experienced qualitative researcher (LTW) conducted the first interview with the honours student interviewer team (RO, KS \& $A J$ ) observing as a form of interviewer training. This interview was also used to obtain data on the timing and feasibility of the interview protocol.

Participants completed the demographic screening survey online, and were contacted by email to arrange an interview time. All interviews were conducted between March and April 2017. After the first interview, subsequent interviews were conducted using a team approach. One interviewer took the lead role and the others acted mainly as observers only speaking to seek clarification or additional information. The team approach was used with the aim of achieving consistency between interviews ${ }^{(25)}$. The role of interviewer was rotated in a sequential order for each interview. The telephone interviews were conducted on loud-speaker and audiorecorded, including recording of participant consent for all aspects of the process, including the presence of the two other team members. Interviews were transcribed verbatim with the removal of vocalised pauses such as 'um' (26). Transcripts were emailed to participants to confirm the information was correct and to allow verification of the data. Four participants responded with amendments that filled gaps where words were missed. Each recording and the subsequent transcript were assigned a participant numerical code and actual names were deleted for anonymity and confidentiality.

Data analysis was conducted concurrently to data collection, using an inductive thematic approach to identify, analyse and report patterns in the collected data ${ }^{(24)}$. The first three interviews were coded individually by all three interviewers and then the codes discussed as a team to established coding consistency. All subsequent interviews were coded individually and cross-referenced in duplicate. If coding inconsistencies arose, a third researcher was involved. The original transcript was reviewed for context and discussed until consensus was reached. All authors worked together to group codes in to subthemes based on similarity, which were subsequently grouped in to themes ${ }^{(24)}$. The interviewers met with the experienced qualitative researcher after the first three interviews were coded, and weekly thereafter, to enhance the quality, consistency and reliability in the coding technique. Data saturation was reached at 
interview 17, with 111 codes identified ${ }^{(24)}$. However, a further three interviews that had been previously scheduled, were conducted and added to the dataset.

\section{Results}

Twenty dietitians participated in the study ( $\mathrm{n}=17$ female). Duration of experience in the primary care setting ranged from 0.7 to 23 years, with an average of eight years. Participants were located in three Australian states: New South Wales $(n=8)$, Queensland $(n=8)$ and Victoria $(n=4)$. Three-quarters of participants worked part-time and the remaining worked on a fulltime basis. Referrals regarding chronic disease management (e.g Type 2 diabetes mellitus, cardiovascular disease, fatty liver disease, chronic kidney disease) accounted for over half of all referrals for most participants, with services also provided for weight management, gastroenterology, pediatrics, eating disorders, malnutrition, prenatal care and bariatrics. Interviews were between 18-65 minute duration. Three themes, each with subthemes, emerged from the analysis. These are outlined in Table 1 and discussed below.

\section{Theme One: Efficiency and effectiveness are important but there is tension between the two}

Participants had varying definitions of efficiency but they were all related to time, most often in terms of minimising time spent on tasks. Participants sought efficiency in order to maximise the amount of workload completed in a certain period of time, a concept known as productivity. Participants described time management as being crucial to achieving efficiency in practice, with one participant describing 'Time management...I'm getting to a certain level of a consultation by a certain time' (P18, male, 8 years in primary care). Another participant described efficiency in terms of the ability to be effective within a time period:

'There's obviously delivering consults in a timely manner...but also, being able to affect the changes in a reasonable time frame, I'd say that's efficient' (P2, female, 4 years in primary care)

Efficiency through adhering to scheduled session times was seen as important for practitioner satisfaction by one dietitian owner of a practice: 'But I also believe that efficiency for my dietitians means that they are enjoying their work better' ( $\mathrm{P} 5$, female, 8 years in primary care). 
Client outcomes and achieving positive behaviour change were components of definitions of effectiveness.

'As a clinician, I'm thinking about patient outcomes, what is it that the patients want to see, what is it that I want to see and what doctors or other health professionals are after' (P13, female, 12 years in primary care)

Participants who identified themselves as business owners integrated aspects of business productivity such as repeat business, into their definitions of effectiveness.

'This is quite subjective but the repeat referrals that I get from particular GPs is usually a good indication of whether they value the input that we've had' (P10, female, 2 years in primary care)

Some participants expressed effectiveness as potentially influencing the way that other health professionals, funding bodies, and clients perceive dietitians.

'Well it's vitally important because it gives you meaning and reasoning for your professional input. If you can't measure outcomes, then how can you economically rationalise your professional input and successfully argue your worth or value as a professional?' (P9, female, 15 years in primary care)

However, several participants described a tension between being able to achieve efficiency whilst maintaining effectiveness in their client interactions. While efficiency was considered important, these participants tended to favour effectiveness, given the impact on client care.

'You shouldn't affect your effectiveness when you're being efficient. I don't want to see that the quality of service is less when someone is being efficient' (P5, female, 8 years in primary care)

Favouring efficiency was perceived to reduce the quality and effectiveness of client care. Some participants resorted to increasing consultation length to be able to meet client needs, thus favouring effectiveness and sacrificing efficiency. It was recognised that favouring effectiveness through longer (therefore less financially productive) consultation times as reduced business profitability or impinging on personal time:

'I'm selling my time, and that's my income. Am I just seeing clients who, you know, for two hours, when really, I'm only charging for one hour' (P10, female, 2 years in primary care). 
'Some clients that I saw were incredibly complicated and I didn't leave that normal time that I leave at the end of a consult to do that. So, I put the clients' needs ahead of my own time off work' (P16, female, 10 years in primary care)

\section{Theme Two: Efficiency and effectiveness are influenced by personal and structural factors}

Practitioners perceived several barriers to their being able to achieve efficiency and effectiveness including practitioner characteristics, structural barriers and client characteristics. Some dietitians recognised that their length of experience in primary care affected their efficiency in terms of time spent completing administrative tasks such as report writing and writing letters to the General Practitioner.

'Experience. As your confidence grows and as your skills grow, I think you become more efficient at getting to the core issues, you're not worrying so much about all the details that may be irrelevant' (P20, female, 11 years in primary care)

Another dietitian factor impacting efficiency was practitioner wellbeing. This included factors such as sleep and mind set, both perceived to impact the time and quality of consultations. Keeping energy levels up by the frequency of food intake was seen as important by this participant:

'Another really important thing to being efficient is getting to eat lunch... and that impacts my own physical health, and obviously that also impacts my afternoon clients because I'm not fuelled up ready to smash the afternoon out and give really good clientcentred-focus consults' (P18, male, 8 years in primary care)

Several participants acknowledged structural barriers that impede their ability to achieve efficient and effective practice. One practitioner, whose primary clientele were funded through Medicare (the health funding agency of the Australian government), described how the prescribed amount of financial remuneration for clients (which is around half the amount typically charged for initial consultations) limited the time available for the consultation, which in turn impacted effectiveness:

'If we had more money, we could spend more time with the client. Which would mean we could get better time, better understanding of the client, better measure of results, better effectiveness' (P18, male, 8 years in primary care) 
211 Time and financial constraints were reported as barriers to implementing time saving technology by some participants. As one participant said: 'I would much prefer to use Medical Objects [electronic health records] but it's much more expensive than just faxing it as a small business owner' (P5). Another participant saw technologies as costly, but perceived it as an investment for the financial gain of increased client numbers.

'...technology is going to be make or break for private practice dietitians because without it you simply don't make enough money to survive' (P8, female, 8 years in primary care)

Some practitioners worked in locations that brought other barriers to being efficient.

'Sometimes I run into trouble with where my room is situated with bad internet connection ... it doesn't allow me to process things immediately and I have to do it later' ( $\mathrm{P} 1$, female, 1.5 years in primary care)

Client behaviours were seen as influencing the efficiency of practice. Clients cancelling appointments or attending appointments late were factors identified by participants as compromising efficiency and productivity in primary care. One participant described the risk of time wasted due to non-attendance.

'Even actually DNAs [Do Not Attends]...that's something that decreases our efficiency because then we will be sitting there for half an hour and there's no patient' (P15, female, 3 years in primary care)

Client behaviours inside the consultation such as distractions, level of client comprehension, as well as the complexity of the referral case, were seen to influence efficiency.

'If the patient is distracted... if they have their kids or whatever there that usually isn't an efficient consult because I don't usually get done what I wanted to get done' (P6, female, 0.7 years in primary care)

'Depending on the actual patient themselves and their level of knowledge that really impacts on your efficiency as well because they might take a little bit longer' (P11, female, 2.5 years in primary care) 
Several participants were actively seeking strategies to improve the efficiency and effectiveness of dietetic service in primary care. Support from health professionals and other dietitians in the form of feedback and advice for future improvement, was sought with the aim of improving practice.

'We need to be more supportive of each other within the profession. Support

However, the initial financial impact of hiring administrative support was considered a barrier by one participant: analysis.

'If I had extra people working for me then obviously I wouldn't have to deal with answering the phone, making bookings, taking payments so I could fit more clients in... I don't earn enough to pay anyone to do all that' (P12, female, 5 years in primary care) Technology was viewed as having a role to play in reducing time spent on data collection, analysis and client education during consultations. Some participants also reported utilising apps and audio-visual media to provide nutrition education to clients, saving time during the face to face component of the consultation.

'Well I think it's going to be an efficient way of educating people is to actually use that sort of audio-visual medium... I see increasing my efficiency as people being educated in their own time' (P4, male, 10 years in primary care)

'Having the nutritional assessment done before I see the client saves a lot of time during the consultation' ( $\mathrm{P} 9$, female, 15 years in primary care). 

'...Rather than writing out a food diary for some of my patients, I am actually typing directly into this software system (Nutritics) as they tell me what they eat and it gives me a nutritional breakdown of that food' (P5, female, 8 years in primary care)

Not all participants felt this way. Some reported that they preferred collecting data within the consultation time itself to enhance validity of the information. These practitioners felt that clients completing tasks prior to consultations increased the risk of inaccuracy and noncompletion, therefore resulting in unexpected extra time spent within the consult to collect more accurate information.

'Coming to see me for first time and they say "Agh sorry I forgot to complete the prequestionnaire" that means we have to go from scratch and getting to know this patient' (P\&, female, 4 years in primary care)

\section{Discussion}

This pragmatist study was the first to qualitatively explore dietitians' perceptions of the constructs of efficiency and effectiveness in the primary care setting. These factors are likely to be of importance given the growing number of primary care dietitians and the significance of primary health care in providing first point-of-care to individuals ${ }^{(7,27)}$. Dietitians in the present study defined efficiency predominantly in terms of time as a measure of productivity. Tools used to enhance efficiency were those that saved time spent on administrative and data collection tasks, so that more time could be devoted to counselling the client. Time spent face to face was privileged above time spent in other tasks, presumably because the face-to-face time was seen as engendering the outcomes. This is an interesting finding, especially given it is counter to the trend towards technology delivered services, but perhaps to be expected in a profession based on counselling ${ }^{(28)}$. Effectiveness was a highly valued aspect of primary care, perhaps even more highly valued than efficiency, because it was seen as client-focused, establishing a tension between these two important constructs. A balance of efficiency in managing their business and effectiveness in achieving client outcomes was seen as the ideal however it was rarely achieved.

Given primary care dietitians are paid per consultation rather than according to time worked, time was seen by some participants as a constraint that could negatively impact client care. The risks to effectiveness of time constraints supports the findings of other studies suggesting that 
sacrificing consultation time for financial remuneration impedes the perception of quality and effectiveness of the nutrition care provided ${ }^{(7-9,14)}$. Reducing consultation time in line with the level of state funded remuneration in Australia may therefore prevent optimal client care ${ }^{(11,29,}$ 30). Given that state-funded clients comprise $40 \%$ of the load for the average primary care dietitian, managing this client base efficiently is vital for business sustainability ${ }^{(9,21)}$. In managing a private practice, dietitians need to balance having sufficient client numbers to maximise remuneration, while still providing an effective service to individuals. If too many clients are seen for less than the optimal time period to enhance efficiency, the likelihood of clients returning for subsequent visits is reduced. Conversely, consulting a smaller clientele provides less financial sustainability. Both a strong client base and financial stability are required for business sustainability in the primary care setting ${ }^{(7)}$.

Dietitians in the present study were aware of the need to balance efficiency and effectiveness for business sustainability and job satisfaction, but cited barriers to the investment of time and finances necessary to achieve this balance. Technology was seen by some participants as a means to reduce time spent on consultation tasks such as data collection, data analysis and client education, allowing for greater client-focused time, and therefore effectiveness, within consultations. The perceived benefits of improving data collection, analysis and client education though the aid of technology has been found in other studies ${ }^{(31,32)}$. Technological aids such as data entry programs, personal digital assistants and the ability to access the internet through mobile devices allows clients to input background information and their diet history in their own time, leaving more consultation time for nutrition education and goal setting ${ }^{(31)}$. This present study demonstrates that while primary care practitioners were aware of these technologies, lack of familiarity with programs, costs and time taken to learn new systems were perceived as potential barriers to their use in this sector of the workforce.

Administrative assistance was perceived as a potential contributor to workplace efficiency. Audit studies have shown that time spent before, during and after each consultation by dietitians is significantly reduced (42 minutes compared to 54 minutes) for dietitians who have the support of administrative assistants managing tasks such as emails, bookings and payments $(7,9,21)$. Dietitians with administrative support have been found to charge similar fees to those without that support ${ }^{(21)}$. One participant in the present study described a growth in client numbers through the employment of an administrative assistant. Cost benefit studies considering the financial cost and value of hiring an administrative assistant are needed to further address this question. 
Dietitians in the present study perceived that the ability to balance efficiency and effectiveness was something that grew with experience or through increased support from other primary care dietitians. Previous research has found that while some dietitians are content to work in isolation, most feel that support from other dietitians is important for the growth of individuals and the profession ${ }^{(8,33)}$. Working in a multidisciplinary team has previously been found to improve practice through (i) more appropriate referrals, (ii) increased support for areas outside of dietitians' scope of practice and (iii) opportunities to facilitate consistent nutrition messages by practice teams $(14,34)$. These strategies are thought to result in better client outcomes, improved quality of care and client safety ${ }^{(35)}$. Therefore, enhancing supportive networks with other dietitians and health professionals may contribute to continuity of care and reducing workload to improve the efficiency and effectiveness of all professionals in the primary health care team.

A limitation of the present study was that the data was collected by three interviewers which may have impacted the consistency of the data collected. The steps taken to limit this included use of an interview protocol, the three interviewers all being trained together by an experienced qualitative researcher, and all three interviewers marking in a team and being present at every interview. This study was combined with one on the use of technology in the same target group with the aim of maximising participation and minimising participant burden. We acknowledge this may have influenced responses to be more technology-focused, but attempted to limit this potential bias by exploring technology at the end of the interview protocol. The themes touching on technology reported in this paper arose in response to the effectiveness and efficiency questions of the interview. Despite the national recruitment strategies used, all participants were located within the eastern Australian states. However, this is reflective of the distribution pattern of the primary care dietetic workforce in Australia with the majority being located in the eastern states ${ }^{(36)}$. Given the study is qualitative, with recruitment primarily by convenience, the sample is not representative, and the findings would ideally now be quantified in a national study with a representative sample of primary care dietitians. The views and attitudes revealed in this study highlight the tension faced by primary care practitioners in trying to achieve a balance between efficiency and effectiveness. This has implications for resource utilisation and time management for practitioners in primary care under a private practice model, and therefore for workforce sustainability. Improving efficiency to enhance business sustainability is a concept not limited to the dietetic profession, or to those in 
Australia, with potential relevance to other allied health professionals working in primary care around the globe.

\section{Transparency Declaration}

The lead author affirms that this manuscript is an honest, accurate, and transparent account of the study being reported. The lead author affirms that no important aspects of the study have been omitted and that any discrepancies from the study as planned have been explained.

\section{References}

1 Howatson A, Wall CR, Turner-Benny P (2015) The contribution of dietitians to the primary health care workforce. J Prim Health Care. 7, 324-32.

2 Mitchell LJ, Ball LE, Ross LJ et al. (2017) Effectiveness of Dietetic Consultations in Primary Health Care: A Systematic Review of Randomized Controlled Trials. J Acad Nutr Diet. 117,1941-62.

3 Robbins JM, Thatcher GE, Webb DA, et al. (2008) Nutritionist visits, diabetes classes, and hospitalization rates and charges - The Urban Diabetes Study. Diabetes Care. 31, 655-60.

4 Centers for Disease Control and Prevention (2017) Chronic Diseases: The leading causes of death and disability in the United States. https://www.cdc.gov/chronicdisease/overview/index.htm (accessed March 2018).

5 The Australian Government Department of Health (2017) Chronic Conditions. http://www.health.gov.au/internet/main/publishing.nsf/content/chronic-disease (accessed March 2018).

6 Department of Health and Social Care (2012) Long Term Conditions Compendium of Information: Third Edition. https://www.gov.uk/government/publications/long-termconditions-compendium-of-information-third-edition (accessed April 2018)

7 Ball L, Larsson R, Gerathy R, et al. (2013) Working profile of Australian private practice Accredited Practising Dietitians. Nutr Diet. 70, 196-205.

8 Harper C, Maher J (2017) Investigating Philosophies Underpinning Dietetic Private Practice. Behav Sci. 7, 11. 
9 Cant RP (2010) Today's profession: views and practice of private practice dietitians re Medicare Chronic Disease Management Program. Nutr Diet. 67, 77-83.

39510 Stein K (2016) Propelling the Profession with Outcomes and Evidence: Building a 396 Robust Research Agenda at the Academy. J Acad Nutr Diet. 116, 1014-30.

39711 Sladdin I, Ball L, Bull C et al. (2017) Patient-centred care to improve dietetic 398 practice: an integrative review. J Hum Nutr Diet. 30, 453-70.

39912 Cant RP, Aroni RA (2008) Exploring dietitians' verbal and nonverbal communication 400 skills for effective dietitian-patient communication. J Hum Nutr Diet. 21, 502-11.

40113 Lu AH, Dollahite J (2010) Assessment of dietitians' nutrition counselling self-efficacy 402 and its positive relationship with reported skill usage. J Hum Nutr Diet. 23, 144-53.

40314 Hancock RE, Bonner G, Hollingdale R et al. (2012) 'If you listen to me properly, I 404 feel good': a qualitative examination of patient experiences of dietetic consultations. J Hum 405 Nutr Diet. 25, 275-84.

40615 Cant RP (2009) Communication competence within dietetics: dietitians' and clients' 407 views about the unspoken dialogue--the impact of personal presentation. J Hum Nutr Diet. $408 \quad 22,504-10$.

40916 Oxford Dictionaries (2018) Effectiveness. Oxford University Press. 410 https://en.oxforddictionaries.com/definition/effectiveness (accessed April 2017).

41117 Alaghemandan H, Yarmohammadian MH, Khorasani E et al. (2014) Efficiency 412 improvement of dentistry clinics: introducing an intervening package for dentistry clinics, 413 isfahan, iran. Int J Prev Med. 5, 176-84.

41418 Jortberg BT, Fleming MO (2014) Registered dietitian nutritionists bring value to 415 emerging health care delivery models. J Acad Nutr Diet. 114, 2017-22.

41619 Jansen S, Ball L, Lowe C (2015) Impact of the Medicare Chronic Disease 417 Management program on the conduct of Australian dietitians' private practices. Aust Health 418 Rev. 39, 183-9.

41920 Brown LJ, Mitchell LJ, Williams LT et al.(2011) Private practice in rural areas: An 420 untapped opportunity for dietitians. Aust J Rural Health. 19(4),191-6. 
$42121 \quad$ Brown JA, Lee P, Ball L (2016) Time and financial outcomes of private practice 422 dietitians providing care under the Australian Medicare program: A longitudinal, exploratory study. Nutr \& Diet. 73, 296-302.

42422 Cornwell P, Foster M, Tweedy S et al. (2009) Better than nothing? Restrictions and 425 realities of enhanced primary care for allied health practitioners. Aust J Prim Health. 15, 32642634.

42723 Glasgow RE (2013) What does it mean to be pragmatic? Pragmatic methods, measures, and models to facilitate research translation. Health Educ Behav 40, 257-265.

42924 Liamputtong P, Ebooks C (2013) Research methods in health: foundations for 430 evidence-based practice. South Melbourne, Victoria, Australia: Oxford University Press.

43125 Musselwhite K, Cuff L, McGregor L et al. (2007) The telephone interview is an 432 effective method of data collection in clinical nursing research: A discussion paper. Int $J$ 433 Nurs Stud. 44, 1064-1070.

43426 Gillham B (2005) Research Interviewing: The Range of Techniques. UK: Open 435 University Press

43627 The Australian Government Department of Health (2013) Primary health care in 437 Australia. http://www.health.gov.au/internet/publications/publishing.nsf/Content/NPHC438 Strategic-Framework phc-australia (accessed March 2017).

43928 Ball L, Eley DS, Desbrow B et al. (2016) Association between dietitians' personality 440 profiles and practice areas. Nutr Diet. 73, 247-253.

44129 Whitehead K (2015) Changing dietary behaviour: the role and development of 442 practitioner communication. Proc Nutr Soc. 74, 177-84.

$443 \quad 30$ Cant R, Aroni R (2007) Melbourne dietitians' experience of Medicare policy on allied 444 health services (strengthening Medicare; Enhanced Primary Care) in the first 12 months. Nutr 445 Diet. 64, 43-49.

44631 Probst Y (2011) Dietitians in the electronic age: Progressing towards e-health. Nutr $447 \quad$ Diet. 68, 177-78.

44832 Rollo ME, Hutchesson MJ, Burrows TL et al. (2015) Video Consultations and Virtual 449 Nutrition Care for Weight Management. J Acad Nutr Diet. 115, 1213-25. 
45033 Beckingsale L, Fairbairn K, Morris C (2016) 'Two working together is so much better 451 than just one': Professional support needs of primary healthcare dietitians: Professional 452 support needs of primary health-care dietitians. Nutr Diet. 73, 220-28.

$453 \quad 34 \quad$ Beckingsale L, Fairbairn K, Morris C (2016) Integrating dietitians into primary 454 health care: benefits for patients, dietitians and the general practice team. J Prim Health 455 Care. 8, 372-80.

45635 Sharp M (2006) Enhancing Interdisciplinary Collaboration :In Primary Health Care. 457 Can J Diet Pract Res. 67, S4-S8.

$45836 \quad$ Health Workforce Australia (2014) Australia's Health Workforce Series - Dietitians 459 in Focus. 